\title{
Inmanencia, intencionalidad y representación en Tomás de Aquino
}

\author{
PATRICIA MOYA CAÑAS \\ Universidad de los Andes (Chile) \\ pmoya@uandes.cl
}

\begin{abstract}
Resumen
El artículo se propone rehabilitar el concepto tomasiano de representación cognoscitiva que es fuertemente criticado en su versión post cartesiana. Con este objetivo se contextualiza la representación en el marco más amplio de otras características que Tomás de Aquino atribuye al conocimiento humano, concretamente las de inmanencia e intencionalidad. El análisis de estos atributos del conocimiento permite establecer el ámbito propio del acto cognoscitivo, diferenciándolo de una condición física o natural.
\end{abstract}

Palabras claves: Tomás de Aquino, conocimiento, representación, inmanencia, intencionalidad.

\section{Immanence, Intentionality and Representation in Thomas Aquinas}

\begin{abstract}
This article aims at recovering Aquinas' concept of cognitive representation which is strongly criticized in its post-cartesian sense. For this purpose, representation is contextualized in the wider frame of other characteristics that Thomas Aquinas attributes to human knowledge, i.e. that of immanence and intentionality. The analysis of these features allows to establish the proper scope of the cognitive act and to differentiate it from a physical or natural condition.
\end{abstract}

Key words: Thomas Aquinas, knowledge, representation, immanence, intentionality.

Licenciada en Filosofía por la Universidad Católica de Chile. Doctora en Filosofía por la Universidad de Navarra (España). Actualmente es profesora asociada e investigadora en el Instituto de Filosofía de la Universidad de los Andes (Santiago de Chile). Entre sus publicaciones destacan los libros El principio del conocimiento en Tomás de Aquino (1994) y La intencionalidad como elemento clave en la gnoseología del Aquinate (2000). 


\section{Introducción}

Desde diferentes ámbitos de la filosofía contemporánea se ha realizado una severa crítica al representacionismo gnoseológico. Heidegger caracteriza la Edad Moderna como la «época de la imagen del mundo» en la que la verdad se sustituye por la certeza de la representación. Descartes, particularmente, inaugura esta forma de filosofar en la que el ente se pone ante el sujeto quien en esta referencia a sí mismo le impone sus normas: «El hombre se convierte en el representante de lo ente, en el sentido objetivo» (Heidegger, 2000: 75). El pensamiento deviene representación como una forma de asegurar lo conocido: «El re-presentar es una objetivación dominadora que rige por adelantado» (Heidegger, 2000: 87).

Más cercano en el tiempo, Rorty es un referente obligado en la discusión contemporánea de la representación, especialmente en Philosophy and the Mirror of Nature. Estableciendo las debidas diferencias históricas, señala Rorty que Aristóteles y Tomás de Aquino comparten con gran parte de la tradición filosófica la división entre aquello que pertenece a un ámbito exterior - el cuerpo- y a otro interior - el alma o la mente (Rorty, 1979: Part One, Chapter II). De esta división surge necesariamente la comprensión del conocimiento como representación. En la filosofía de Tomás de Aquino, la representación está fundamentada desde una perspectiva metafísica: la capacidad de intuiciones, conceptualizaciones o categorizaciones preexistentes al desarrollo propiamente científico sostiene el desarrollo ulterior del conocimiento entendido siempre como representación (Rorty, 1979: 258).

La representación ha caído en descrédito en nuestra cultura filosófica porque efectivamente induce a concebir el conocimiento como el efecto causal de un objeto externo sobre nuestra conciencia o como una reproducción elaborada por parte del sujeto. En ambos casos se requiere una garantía que certifique la correspondencia entre la causa externa y el efecto interno o entre la reproducción y el original y ¿de dónde la podemos obtener? Nos encontramos así, más bien, ante un obstáculo que una explicación del conocimiento.

El tema de la representación aparece prácticamente en toda la obra de Tomás de Aquino, pero de un modo más expreso en dos obras tempranas: en el Comentario al libro de las Sentencias de Pedro Lombardo y en la Cuestión Disputada De Veritate. En la Suma Teológica utiliza más la 
expresión semejanza (similitudo) ${ }^{1}$, y también la de species intelligibilis, que tiene relación con la de semejanza.

La filosofía del conocimiento tomasiana se desarrolla en gran parte, aunque no exclusivamente, a partir de la tradición aristotélica, y en ella se inspira para el concepto de representación ${ }^{2}$. Con este término Tomás de Aquino introduce una instancia propia del acto cognoscitivo. Es decir, le sirve para expresar que no hay una homogeneidad de orden natural o físico entre el objeto tal como es en sí mismo y tal como es tenido por el sujeto en cuanto conocido. La posesión cognoscitiva es el fruto o resultado de una operación del sujeto por la que éste se identifica cognoscitivamente con lo conocido. No deviene lo conocido, sino que lo posee en un orden diferente al natural o físico, que llama orden intencional.

Esta explicación es coherente con la afirmación aristotélica que por el conocimiento no tenemos en el alma la cosa misma, sino su forma. Afirmación que se basa, a su vez, en la teoría de la identidad cognoscitiva por la que los sentidos al conocer son en acto lo sensible, y el entendimiento al conocer es en acto lo inteligible. Esta identidad no es absoluta, en el sentido que suponga una transformación por parte del sujeto, sino que se realiza solamente en el orden de la potencia o capacidad que tienen las facultades cognoscitivas de hacerse uno con los objetos a los que se ordenan (Aristóteles, 1988: III, 8, 431b 20ss).

Según Tomás de Aquino, la actividad u operación cognoscitiva otorga una cierta presencia a lo conocido en el sujeto cognoscente. La cuestión que se plantea es si se aplica a esta concepción del conocimiento la crítica más bien dirigida al representacionismo moderno, porque efectivamente hay en la explicación tomasiana un uso de la representación cognoscitiva. ¿Consigue Tomás de Aquino superar la dicotomía interno-externo? Su enfoque cimplica la presencia de una entidad intermedia, propiedad de la mente, una suerte de copia o internalización de una realidad exterior en la mente? El objetivo de este artículo es intentar precisar el concepto de representación al interior del pensamiento de Tomás. Para esto se procederá explicando, en primer lugar, el conocimiento como operación inmanente, y luego la intencionalidad cognoscitiva, entendida en su doble aspecto de posesión y referencia. Me parece que este es el marco adecuado para responder,

1 Ver en Tomás de Aquino (1988: I, 59, 2; I, 17, 3; I, 76, 2, ad 4) el uso del término semejanza como aquello que es poseído cognoscitivamente, a diferencia de la posesión de la propia forma.

2 Aristóteles (1988: III, 4, 429a 27-29; 8, 431b 21; 8, 432a 2-4) considera el alma como el lugar de las formas, al intelecto como forma de formas y la capacidad del alma de ser, en cierto modo, todas las cosas. 
intentando seguir la mente de Tomás de Aquino, al problema sobre la discutida representación cognoscitiva.

\section{E1 conocimiento como operación inmanente}

La actividad natural del ser vivo se manifiesta o expresa en las capacidades que le corresponden según su grado o nivel de vida. Aristóteles clasificó estos niveles en vida vegetativa, sensitiva e intelectiva: «El alma es aquello por lo que vivimos, sentimos y razonamos primaria y radicalmente» (Aristóteles, 1988: II, 2, 414a 13-14). La perfección del ser vivo se realiza en el ejercicio de sus facultades, con ello se ordena a su acabamiento, entendido como actualización. En este contexto, Aristóteles comprendió el conocimiento como una expresión de la forma de vida más perfecta. Tomás de Aquino hace suya esta doctrina aristotélica al considerar el conocimiento como una perfección que le corresponde al sujeto por su forma, pues el alma intelectiva es la forma del hombre. Desde esta perspectiva, el conocer no se añade al ser ya constituido como sustancia, sino que es una perfección que lo constituye como tal, es su actualidad. Una característica de esta perfección humana es la infinita apertura del cognoscente a todo aquello que es que remedia, en cierto modo, la limitación de la existencia finita de los seres creados. Los seres que tienen conocimiento se diferencian de los que no tienen en que, además de su propia forma, pueden poseer las formas de otras cosas, tienen así una amplitud perfectiva ilimitada (Aquino, 1988: I, 14, 1) . $^{3}$

Siguiendo una explicación de la Metafísica de Aristóteles (1990: IX, 6, 1048b 18-35), Tomás de Aquino distingue entre las acciones transitivas y las inmanentes. Las primeras obtienen su acabamiento o realización en un producto exterior al sujeto que la realiza; en cambio en las segundas la perfección de la acción redunda en el sujeto que la ejecuta, tal es el caso del sentir, conocer y querer (Aquino, 1988: I, 54, 2). Así se explica que la realización del acto de entender es el entender mismo pues no hay una perfección ulterior, sino la propia del acto (en términos aristotélicos energeia). Esta concepción de la inmanencia operativa permite

3 Similares afirmaciones (Aquino 1988: I, 59, 2 y I, 80) aluden a la capacidad del cognoscente de hacer suya la realidad que le circunda. Idea que obviamente remite a Aristóteles (1988: III, 8, 432a 2-4). Ver también Aquino (1975-1976: 2, 2 y 10, 4, 1). Cf. Dewan (1984) quien hace ver la necesidad de considerar seriamente la capacidad cognoscitiva de la persona humana para comprender su modo de ser propio. El inteligir es un acto que presupone el acto de ser y por esta razón depende en su perfección y actualidad del ser en el que inhiere la facultad intelectiva. 
comprender el modo en que lo conocido existe en el cognoscente. No existe como una producción del que conoce; tampoco está más allá del que conoce, como podría interpretarse en las ideas platónicas. Más bien existe como tenido o poseído por el sujeto en un acto perfectivo que es la realización o actualización de la potencia cognoscitiva. Aristóteles (1988: II, 5, 417a 22-417b) lo ejemplifica con las diversas situaciones en que se puede encontrar un sabio: puede poseer actualmente un conocimiento, cuando está conociendo de una manera explícita o puede poseerlo de manera habitual, en el caso de que posea lo conocido, pero que no lo esté considerando en acto.

La inmanencia propia del acto cognoscitivo fija la atención en la operación del sujeto y considera la pasión o inmutación por parte del objeto como una condición del acto de conocer, pero no como su explicación. Lo entendido en acto no existe en sí como una realidad. Lo real es la cosa existente que es potencialmente inteligible y se vuelve entendida en acto por la operación del entendimiento que conoce. Esta explicación hace indispensable el acto de conocer, porque no basta con el acto de ser propio de la cosa para que ésta se conozca, sino que es necesaria la operación que hace inteligible en acto aquello que por sí mismo solo es potencialmente inteligible.

Es así que lo propio de la inmanencia cognoscitiva es la perfección o actualización de la potencia cognoscitiva en el acto mismo del conocimiento. Afirmar que en el acto de conocer se posee lo conocido es otra manera de decir que lo conocido sólo es tal como término del acto cognoscitivo. La operación cognoscitiva no causa lo conocido, es coactual con él. Se puede decir que lo conocido (en cuanto que conocido) existe por el ser que le confiere el sujeto cognoscente en su operación cognoscitiva. La perfección del conocimiento no proviene exclusivamente de la cosa conocida (proviene de ella en cuanto es su fuente y su objeto), sino que proviene fundamentalmente del acto del sujeto capaz de hacer conocida la cosa existente fuera de la mente. Tomás expresa esta idea en el siguiente texto: «Tampoco el acto de conocimiento se relaciona con su objeto del mismo modo que con la potencia cognoscitiva; en efecto es sostenido en su ser por medio de la potencia cognoscitiva y no mediante el objeto, puesto que el acto está en la potencia misma, pero no en el objeto» (Aquino, 1975-1976: 2, 5, ad 16).

Se ha dicho que la inmutación de la potencia cognoscitiva por parte de la cosa conocida es una condición del conocimiento. Esta afirmación se entiende al considerar que el hombre no es su conocer, sino que hay una distinción entre su ser y su operar. Para realizar el acto cognoscitivo la potencia o facultad debe ser inmutada porque el sujeto no tiene en sí 
mismo las esencias de las cosas. El conocimiento tiene su origen en la realidad que circunda al sujeto y que se le hace presente en la experiencia, entendida en un sentido amplio. A partir de este contacto experiencial, el entendimiento puede realizar al acto cognoscitivo que consiste en la coactualidad entre el entendimiento y el objeto conocido (Aquino, 1988: I,

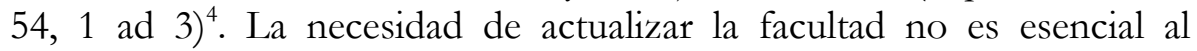
conocer mismo, sino que es propia de la persona humana que conoce a partir de aquello que existe fuera de su inteligencia ${ }^{5}$.

La relación que se establece entre el sujeto cognoscente y el objeto conocido es del todo particular. Mientras que las sustancias, es decir, las realidades materiales o extramentales, tienen una existencia independiente de la facultad cognoscitiva, al ponerse en relación con esta facultad, pierden su independencia, pues se vuelven sensibles $\mathrm{O}$ inteligibles con respecto a ella. La dependencia que adquieren respecto a la facultad no supone un relativismo, sino la adquisición de una forma nueva de relación, que es la cognoscitiva (Vigo, 1995: 281) ${ }^{6}$.

El ser cognoscente y el ser cognoscible son correlativos e indican una relación que se establece entre ambos, pero no son dos propiedades que ambos términos tengan siempre de un modo actual, sino que esta relación es posterior, si se puede hablar así, a la actualidad primera por la que son en cuanto entes. Se puede decir que la cosa qua cognoscible depende del cognoscente, porque se trata de una potencialidad de la cosa que sólo puede actualizarse al ponerse en relación con la facultad de conocer. Ambos términos de la relación se elevan al acto: el sujeto perfeccionado por la posesión de la forma de la cosa y ésta en cuanto conocida en acto. Las dos potencialidades comparten un mismo acto que

4 Esta actualización de la potencia cognoscitiva se realiza gracias a la acción del intelecto agente que hace actualmente inteligible la forma de la cosa conocida. En Aquino (1998: I, 79, 3-5) se explica la necesidad de un intelecto agente.

5 «Pero hay que tener presente que, a veces, estas especies del objeto están en la facultad cognoscitiva solamente en potencia; y entonces sólo hay un conocimiento potencial; y para que conozca en acto se precisa que la potencia cognoscitiva reciba de hecho la especie. En cambio, si la tiene siempre en acto, puede conocer por ella sin que previamente se produzca mutación o recepción alguna. De lo cual se deduce que ser movido por el objeto no es esencial al que conoce, en cuanto que conoce, sino en cuanto que está en potencia para conocer» (Aquino, 1988: I, 56, 1).

6 Explica Vigo (1995: 281) que «la posición habitual de Aristóteles es justamente que los objetos primarios de percepción o 'sensibles' (vgr. colores, sabores, etc.) no tienen existencia independiente de la percepción, mientras que los 'sustratos', es decir, los objetos materiales que en sentido derivado se denominan 'sensibles' sí pueden existir independiente de ésta». Aristóteles (2008: 6b 32-35) afirma: "Así, el conocimiento se dice conocimiento de lo cognoscible, y lo cognoscible es cognoscible por un conocimiento; o la sensación es sensación de algo sensible, y lo sensible, es sensible por una sensación». 
no clausura la radical apertura del cognoscente, sino que permite una permanente capacidad de actualizaciones sucesivas.

La relación que se produce entre las potencias cognoscitivas de la persona humana y las potencialidades de las sustancias, en cuanto objetos de conocimiento, distingue el acto cognoscitivo de uno meramente natural —en el sentido de físico- porque la operación de las facultades cognoscitivas se realiza en un nivel propio que da lugar al ser original del conocimiento (Llano, 1999: 25). La coactualidad entre el sujeto y el objeto de conocimiento permite establecer una diferencia entre las acciones naturales o físicas y la operación cognoscitiva. Las primeras tienen una dirección agente-paciente, pues una realidad física influye en otra inmutándola. En cambio, en el conocimiento no se da propiamente una relación acción-pasión, ni tampoco una inmutación en sentido estricto, sino, más bien, la actualización de aquello que es cognoscible en la sustancia que se ofrece como objeto para el cognoscente. Por dicha actualización lo conocido es poseído por el cognoscente, existiendo cognoscitiva o intencionalmente en él. Fuera del acto de conocer, el sujeto y la cosa son potencialmente cognoscente y cognoscible, y mantienen la independencia que les confiere la actualidad primera.

La coincidencia se realiza exclusivamente en el ser cognoscitivo y no en la realidad natural o extramental. Es decir, la identidad o co-presencia del acto del sujeto y del acto del objeto conocido comparece en su ser formal o cognoscitivo y no en su ser natural. Por esta misma razón no hay una identidad numérica, sino que cada uno de los términos de la identidad tiene su propia realidad. La operación (energeia) cognoscitiva hace que la cosa aparezca ante el sujeto sub ratione obiecti, es decir, como término de la operación y a la vez como medida de ésta. La existencia de la cosa y del sujeto son condiciones de la coactualidad, pero aquello que provoca, por así decirlo, el que sean coactuales entre sí es la operación cognoscitiva que hace cognoscible en acto la cosa. Lo directamente actualizado es la forma del objeto conocido, de tal manera que es la propia actualidad de la cosa la que aparece como el telos y co-principio del acto. En este sentido la cosa, a través de su forma, actúa como medida del acto cognoscitivo, porque este se realiza al conocer la forma. Se da una conmensuración entre ambos actos, pues el acto de conocer se ajusta al de lo conocido y éste al acto de conocer (Polo, 1987: I, 80-81). El siguiente texto de Tomás de Aquino expresa con claridad esta conmensuración:

El entendimiento en acto es absolutamente, o, lo que es lo mismo, es perfectamente la cosa entendida, según se dice en el libro III del De anima. 
Y esto hay que considerarlo no en el sentido de que la esencia del entendimiento pase a ser la cosa entendida o su especie, sino porque es informado completamente por la especie de la cosa entendida mientras la entiende en acto (Aquino, 1956: 1, 2).

Como ya se ha dicho, en el conocimiento no se tiene la cosa en su ser físico, sino que se posee su principio de inteligibilidad. La res intellecta no es igual a la cosa existente, aunque cognoscitivamente se identifiquen. Es a través de la aprehensión de la forma que se conoce la cosa. Este tema se verá más adelante al hablar de la intencionalidad cognoscitiva.

La comprensión del vínculo que se forja entre el sujeto que conoce y lo conocido aleja la idea del conocimiento como una copia de la realidad en el entendimiento, producto de la acción del objeto. En el acto cognoscitivo es donde se realiza efectivamente el conocimiento. Por esta razón es más propio hablar de conmensuración y coactualidad entre el sujeto y el objeto que describirlo como una inmutación ${ }^{7}$.

En consonancia con lo que hasta aquí se ha explicado, la posesión cognoscitiva no se entiende como una reduplicación de la cosa conocida por su representación en el sujeto, sino como un peculiar incremento de la inteligencia, una actualización y perfección. Este incremento intelectual se sigue del hecho que la potencia está ordenada de suyo a tal actualización, de tal manera que el conocer siempre comporta una perfección del sujeto. La operación inmanente explica el modo en el que concurren la forma esencial $y$ las formas cognoscitivas. La intencionalidad cognoscitiva permite dar cuenta de que esta concurrencia no significa «trasladar» la cosa existente extramentalmente al interior del sujeto cognoscente, sino una posesión formal que remite al ser real.

\section{La intencionalidad cognoscitiva}

Tomás de Aquino no trata de una manera expresa el tema de la intencionalidad cognoscitiva, seguramente porque está haciendo uso de un concepto vigente en su época tal como lo muestran las fuentes

\footnotetext{
7 Polo (1987: I, 30-31) explica que el aspecto pasivo del conocimiento tiene que ver con la facultad que requiere pasar de la potencia al acto. El empirismo se caracteriza por una explicación del conocimiento en términos de agente-paciente y plantea la necesidad de un sentido interno capaz de interpretar las impresiones. Kenny (1989) hace una aguda crítica a esta concepción. También la explicación de los filósofos presocráticos naturalistas, tal como la presenta Aristóteles en el De anima, cae en el error de comprender el conocimiento como un efecto.
} 
lexicográficas (Hamesse \& Portalupi, 1992) ${ }^{8}$. A pesar de que son pocos los textos en que menciona este concepto, autores como Maritain, Verneaux y otros han señalado la relevancia que tiene para la comprensión tomasiana del conocimiento humano. A mi juicio es una característica que guarda estrecha consonancia con lo anteriormente explicado acerca de la inmanencia cognoscitiva y que da luces para una adecuada interpretación del uso que Tomás hace de la representación, alejándola de una reduplicación interior de la realidad'.

Uno de estos textos en los que menciona la intencionalidad es un comentario al De anima II, 12, 424a 18-29 en el que Tomás de Aquino explica que la forma «tiene un modo de ser diferente en el sentido que en la cosa sensible. Pues en la cosa sensible tiene una realidad natural y en el sentido, en cambio, una realidad intencional y espiritual» (Aquino, 1979: lección 23, n. 533). Espiritual significa en este caso inmaterial, como opuesto a un existir natural, que podríamos interpretar como físico. Como sinónimo del ser inmaterial se añade el ser intencional. Sin entrar en detalles del conocimiento sensible al que alude el texto, podríamos decir que por esa posesión intencional el sujeto cognoscente es capaz de identificar o discriminar una cualidad que está dentro de los rangos de su capacidad sensitiva. La captación vista en sí misma es inmaterial en el sentido que es un acto cognoscitivo que surge de una potencia natural del ser vivo, pero no es absolutamente inmaterial en la medida que para que se dé la operación sensitiva se requiere que el órgano sensible sea

\footnotetext{
8 Ver también Busa (1974: Sectio I, vol. 4, págs 478 y 650); Moya (2000).

Dewan (1984) afirma que el esse intentionale no es el foco principal de la ontología de Aquino sobre el conocimiento, sino más bien el esse inmateriale. En este trabajo no he abordado el tema de la inmaterialidad, pero pienso que ambas características del conocimiento se requieren y complementan para su adecuada comprensión. La intencionalidad refuerza la dirección hacia las cosas, aspecto característico de la comprensión del conocimiento tomasiana y que también destaca Dewan. En esta misma línea es interesante y sugerente el artículo de Moser (2001) que rebate la tesis de que la posesión del esse intentionale es aquello que distingue al sujeto cognoscente en cuanto tal de los seres no cognoscentes. Argumenta principalmente a partir de textos en los que Tomás de Aquino utiliza el esse intentionale o la intentio en contextos no cognoscitivos como son la transmisión de la información a través del medio en el caso del conocimiento sensible o la apreciación de la estimativa en el caso de los animales. Se podría tratar más a fondo esta cuestión, pero me limito a comentar que Tomás de Aquino puede hacer un uso amplio de estas expresiones que eran conocidas en su ambiente y respecto de las cuales no establece mayores precisiones. En todo caso, siempre hay una referencia a un contexto de información, que permite un uso análogo de estos términos.
} 
física o corporalmente inmutado. En este sentido el conocimiento sensible está ligado al cuerpo ${ }^{10}$.

Para nuestro objetivo, el texto recién comentado es relevante porque expresa un rasgo de la intencionalidad que es la posesión de la forma de la cosa conocida, pero lo más interesante es que añade un dato con respecto a la posesión inmaterial de la forma: junto con poseerse cognoscitivamente, remite o envía a la cosa conocida. Esta característica es más clara en el siguiente texto de la Suma Teológica:

Un ángel conoce a otro por la especie del otro que hay en su entendimiento, y que difiere del ángel del que es semejanza, no como se diferencia lo material de lo inmaterial, sino como lo natural de lo intencional. En efecto, el ángel es una forma subsistente en su ser natural. En cambio, su especie que está en el entendimiento de otro ángel no lo es, sino que allí sólo tiene ser inteligible (Aquino, 1988: I, 56, 2, ad 3).

Nuevamente se presenta la oposición entre natural e intencional para explicar el modo en que lo conocido está en el cognoscente, pero en este caso la distinción es más neta, porque se trata del conocimiento de un ser inmaterial respecto de otro que también es inmaterial. Así queda más manifiesto el carácter exclusivamente cognoscitivo de esta posesión: «allí sólo tiene ser inteligible», es decir, no hay en esta posesión más que el conocimiento del otro. Tomás de Aquino destaca la relación cognoscitiva que se establece entre ambos ángeles a través de la semejanza que uno posee del otro. Este término indica la posesión intelectual de la forma del ángel conocido que permite a la inteligencia referirse o «intencionar» el ser real de lo conocido. El siguiente texto evidencia el sentido remitente de la intencionalidad cognoscitiva:

La similitud existente en la potencia cognoscitiva no es pues principio del conocimiento de la cosa según el ser que tiene en la potencia cognoscitiva, sino según la relación que tiene con respecto a la cosa conocida; y por eso la cosa es conocida no por el modo que la semejanza de la cosa tiene en el

10 Sobre la inmaterialidad del conocimiento sensible en Tomás de Aquino, ver: Moya (1999); Cohen (1982) quien sostiene la tesis que para Tomás la recepción de las formas sensibles tiene lugar en entidades físicas y es siempre un evento físico. En la última parte de mi artículo discuto esta posición de la cual discrepo. Sorabji (1992) afirma que en Aristóteles la percepción sensible ha de ser entendida de un modo exclusivamente fisiológico y que la captación de formas sin materia es una operación exclusiva del entendimiento. Tampoco suscribo esta postura. Ver también Sanguineti (2005 y 2007) quien sugiere que es más adecuado decir que la sensación es psicosomática, porque así se da cuenta tanto del aspecto físico como del anímico. 
cognoscente, sino por el modo en que la semejanza que existe en el intelecto es representativa de la cosa (Aquino, 1975-1976: 2, 5, ad 17).

Se puede ver con claridad que la intencionalidad cognoscitiva en la mente de Tomás expresa tanto la existencia de lo conocido en el cognoscente como la vinculación, a través del conocimiento, con la cosa existente. Es notable que en este texto, a modo de conclusión, devalúe, en cierto modo, el ser o el existir mental, para darle prioridad al ser cognoscitivo. Se puede decir que es más relevante el representar y remitir a lo conocido que el modo en que la forma está en el cognoscente. Aunque lógicamente ambos aspectos son dos caras de una misma moneda, porque lo conocido no «existe en el sujeto» como si fuese algo, es decir, como si tuviese una realidad semejante a la cosa natural, sino que existe conformando la inteligencia en su haber propio, es decir, en su ser cognoscente (Anscombe \& Geach, 1973: 94-96) ${ }^{11}$. Desde esta perspectiva, la posesión intencional de lo conocido es «simultánea» a la dirección o intención que tiene la inteligencia hacia lo conocido, el ser conocimiento de.

Al explicar la inmanencia cognoscitiva se aludió al carácter relacional del conocimiento. Nuevamente quiero insistir en este aspecto que se destaca más aun con esta otra característica que es la intencionalidad. El vínculo con la realidad conocida, que se adquiere en la posesión formal o intencional de la cosa constituye la finalización de la potencia. Ésta completa su operación en la referencia a lo conocido (Kenny, 1993: $107)^{12}$.

Contribuye a explicar este carácter remitente o referencial de la intencionalidad la explicación de la relación que nos proporciona Tomás de Aquino en la Suma Teológica en la cuestión 28 de la prima pars. En el artículo $1^{\circ}$, en la objeción $4^{a}$ se dice que las relaciones que son consecuencia de la operación del entendimiento son relaciones de razón. Este argumento permite establecer en el corpus del artículo la distinción entre las relaciones reales y las de razón. Son relaciones reales las que se

11 Estos autores hacen ver que ser la sensación o el pensamiento de un X no es una relación en la que la sensación o el pensamiento permanecen constituyéndose como un nuevo «estado mental», sino que más radical y simplemente lo que la sensación o el pensamiento es.

12 Kenny (1993) afirma: «Aquinas' theory of intentionality can be summed up thus: the object of thought exists, intentionally, in the intellect; its existence is the actualization, the life, of the intellects. Ver también Kenny (1984: 71-76), resumidamente su tesis es que Tomás de Aquino sin ser un idealista es un antirealista, porque no piensa que las ideas sean entidades intermedias que representan el mundo, sino que son modificaciones de nuestro intelecto que consisten en una habilidad adquirida de pensar. La única existencia de las ideas es su habilidad de ocurrir o darse en pensamientos. 
encuentran en la misma naturaleza de las cosas, por ejemplo, en la concepción física de entonces, la relación de los cuerpos pesados con el centro. En cambio, son de razón las que se dan cuando el orden indicado por los extremos de la relación se encuentran sólo en la aprehensión que hace la razón, por ejemplo, la comparación del hombre al animal como la especie al género. En la respuesta a la objeción señalada se precisa más la cuestión respecto al tema que nos ocupa. Se explica que las relaciones que son consecuencia sólo de la operación del entendimiento son relaciones de razón, porque el entendimiento las establece entre dos cosas conocidas, por ejemplo, el vínculo entre género y especie. Pero no sucede lo mismo con relaciones que son consecuencia de la operación del entendimiento cuando este concibe y conoce. Aquello que concibe establece una relación real con lo concebido. No es lo mismo considerar una relación en cuanto captada por el entendimiento - que no es en este caso uno de los términos de la relación, sino la facultad que los captaque considerar el entendimiento como uno de los términos y establecer así una relación real entre el acto cognoscitivo y aquello que es conocido. En este caso se considera la realidad del acto y no la relación lógica. Es algo que acontece al entendimiento que se vincula realmente con el término que es lo conocido. Esta perspectiva sólo puede ser captada si se analiza el conocimiento en su ser real en el sujeto, desde la perspectiva de la primera persona, pues visto en el objeto sólo atenderíamos a la relación lógica ${ }^{13}$.

El acto cognoscitivo tiene su razón de ser en este referirse a la realidad tanto en lo que podríamos llamar la incoación del acto, como en su plena realización. El ser intencional sólo es posible en relación a un ser real, existente por sí mismo del cual la intentio es la expresión del carácter conocido de dicho ser. Este ser conocido se diferencia, en términos de Tomás, del ser natural, es decir del ser extramental, usando una expresión poco adecuada, porque no existe un ser mental, sino más bien el ser en cuanto conocido.

Aquí se entiende el conocimiento como un concepto que expresa un logro, o como un acto que tiene un telos, una perfección, es decir, que realmente conoce la cosa. La forma poseída intencionalmente no es diferente a la que posee la cosa conocida, pero en sus rasgos cognoscitivos, es ahí donde se da la identidad que se sigue de la

13 Hubert (1992: 38) afirma: «Ainsi le connaissant et le connu ne participent pas de la même façon à cette existence intentionnelle, on pourrait dire qu'ils ne contribuent pas à part égale à l'existence de la relation du sujet à l'objet. Il n'y a pas d'équivalence entre les termes de cette relation. La relation est réelle du connaissant au connu tandis que du connu au connaissant c'est l'esprit qui établit la relation. Le sujet d'inhérence de cette existence intentionnelle ou de cette relation c'est le connaissant seul, c'est en lui que la relation est réelle». 
coactualidad ya mencionada. Se puede llamar identidad en el sentido de que no hay un plus cognoscitivo o una configuración del objeto por parte del cognoscente, sino que se da exclusivamente el encuentro de los actos en sus respectivas posibilidades de captación y mostración ${ }^{14}$. Hay una objetualización de lo conocido, es decir, a la par que se da la identidad cognoscitiva, se reconoce el carácter de otro del objeto que se conoce. En el conocimiento se llega a ser el otro sin ser otro, es decir, sin modificarse en el propio ser, sin ser alterado, sino sólo perfeccionado. Este hacerse uno con la forma del otro significa que se debe comprender lo otro en su objetualidad. Se puede hablar así de un contenido cognoscitivo: hay algo - lo otro- que es captado y conocido. El ser intencional o ser conocido actúa como representante de la cosa, o, mejor, presenta la cosa en su cognoscibilidad. Esta es la razón o forma bajo la cual el sujeto que conoce accede a la cosa en su ser natural. No hay un contacto inmediato con lo conocido, sino que justamente por ser conocido hay una formulación intelectual que permite el acceso a lo que la cosa es (Llano, 1999: 21). Pero dicha formulación no es ajena a la cosa: «La cosa entendida se identifica con la cosa real por lo que concierne a su haber, es decir, al conjunto articulado de sus notas: a lo que hace que esa forma o esas formas configuren precisamente esta cosa y no otra» $\left(\right.$ Llano, 1999: 128) ${ }^{15}$.

\section{La representación}

Para comprender adecuadamente el sentido que tiene el término «representación» en Tomás de Aquino se necesitaba esclarecer, o al menos intentarlo, el carácter inmanente e intencional del conocimiento. La inmanencia, porque la representación forma parte de la operación cognoscitiva. La intencionalidad, porque es a través de lo representado que se accede a la cosa conocida. El punto clave en este tema es

\footnotetext{
14 Hubert (1992: 41): «Toute l'action du connaissant se termine formellment à cette relation sujetobjet en non à un effect produit immanent au connaissant. C'est pourquoi c'est una action terminale qui est autant qualification qu'activité. La connaissance est dans l'action et non dans un résultat distinct de l'action, car cette action est elle-même résultat au sens où résultat signifie jaillissement (resultatio). La connaissance apparaît ainsi à la fois comme opération et résultat; comme operation du sujet, comme son cuvre, elle est une activité tandis que comme résultat elle est achèvement qualitatif, un perfectionnement du sujets.

15 Llano (1999: 123) dice, al hablar de la identidad que se da entre el entendimiento y la cosa conocida: «De suerte que tales formas no son propiamente 'cosas', sino justamente representaciones, es decir, presentaciones de la esencia de las cosas ante la mente».
} 
nuevamente la distinción entre natural y cognoscitivo. El representar no es natural. No tenemos, en el caso del conocimiento intelectual, imágenes o pequeños idola como pensaban los estoicos, ni tampoco la representación a la que se refiere Tomás es un proceso neurofisiológico. Lo más elemental que quiere decir Tomás con el término representación es que no tenemos la cosa misma, sino aquello que nos la da a conocer a nuestra inteligencia: «según aquello que está representado en el cognoscente y no según aquello que se tiene en el cognoscente como existente» (Aquino, 1975-1976: 2, 5, ad 17).

Representación y semejanza, como dijimos al comienzo del trabajo, son dos términos que el Aquinate usa indistintamente, porque quiere expresar que tenemos en nosotros un conocimiento acorde con lo que la cosa es en su naturaleza. Es decir, al conocer hay una conformidad no con el ser natural de la cosa, sino con la naturaleza de la cosa conocida, la que no puede tener más que una «existencia» mental o representada (Aquino, 1975-1976: 2, 5, ad 5) ${ }^{16}$. Esta «existencia» a la que se alude con la representación se explica por la operación misma del conocimiento que forma aquello que conoce, es decir, lo convierte en objeto del acto cognoscitivo. Debido a que el conocimiento se realiza en una operación inmanente por la que se establece una relación peculiar del sujeto cognoscente con aquello que conoce, la relación intencional, es necesario que haya una mediación entre la operación cognoscitiva y lo que podríamos llamar el acceso intelectual a la cosa conocida. En sentido estricto la representación no es «idéntica» a la cosa, porque no es la cosa, sino nuestro modo de conocerla. Esto quiere decir que captamos intelectualmente los rasgos esenciales de la cosa, los cuales, unidos a nuestra experiencia, entendida en sentido amplio e incluyendo la percepción y nuestro trasfondo cultural, nos permiten conocer. En este punto es necesario advertir que el conocimiento de la esencia de las cosas no es intuitivo, ni siempre fácil de alcanzar, pero ese es el objeto al que apunta nuestra inteligencia.

Volviendo a la identidad entre cognoscente y objeto conocido, ésta tiene que ser entendida en el nivel cognoscitivo, es decir, se da de un modo mediato cuando se logra esta conformidad de naturaleza que se explicó arriba. No siempre se alcanza la identidad, porque la representación puede ser más o menos adecuada, la inteligencia puede

16 Tomás de Aquino distingue en el texto entre una conformidad in natura que sería una semejanza con el ser natural de la cosa y la conformitas naturae, por la que el cognoscente comprende aquello que la cosa es. 
penetrar mejor o peor la naturaleza de la $\operatorname{cosa}^{17}$. El punto importante es que conocer es captar lo que la cosa es. Y según esto, la representación cumple el papel de remitir a la cosa, no constituye el término del conocimiento, sino aquello por lo que el entendimiento accede a la cosa, la comprende.

La mediación representativa guarda estrecha relación con la teoría tomasiana de la species intelligibilis, que no se tratará en este artículo ${ }^{18}$, y que es el modo como el entendimiento es informado para acceder a la cosa, ya que requiere de esta determinación para formar su objeto, es decir, para especificar el contenido de la operación cognoscitiva que de suyo está abierta a todo lo que es. Tomás refiriéndose a la species, afirma: «Pues lo primero que se entiende es la realidad de la cual la species intelligibilis es su semejanza» (Aquino, 1988: I, 85, 2). La operación del entendimiento otorga un estatuto representativo y, con ello, cognoscitivo al contenido o información que proviene de la cosa misma ${ }^{19}$. La representación tomasiana no se entiende como una copia o calco de la cosa, porque media la operación del entendimiento. Tampoco se puede entender como aquello a partir de lo cual se deriva o deduce lo conocido, porque no constituye una «esfera mental» cerrada en sí misma. Se puede decir que el contenido de la representación se desvela en la misma intencionalidad o referencialidad, porque no es conocido en cuanto contenido, sino por una reflexión del entendimiento sobre sí mismo. Como se dijo arriba, lo primero conocido es la cosa. Así dice Tomás:

Y llamo 'intención entendida' a lo que el entendimiento concibe en sí mismo acerca de la cosa entendida. Intención que, en nosotros, no se identifica con la cosa que entendemos ni con la sustancia de nuestro entendimiento, sino que es una cierta semejanza de lo entendido concebida en el entendimiento y expresada por las palabras; por eso, la intención entendida se llama 'verbo interno', que es expresado por el verbo externo. Y se ve ciertamente que dicha intención no se identifica en nosotros con la

\footnotetext{
17 Aquino (1975-1976: 8, 1) afirma: «Y así, si la semejanza es deficiente con respecto a la representación de la especie, no lo es respecto de la representación del género, se conoce aquella cosa según la razón del género y no según la razón de la especie, si también es deficiente con respecto a la representación del género, la representará sólo según una conveniencia de analogía; entonces tampoco será conocida según la razón del género, así como si se conociera la sustancia por la similitud del accidente».

18 Para el tema de la species intelligibilis, ver Aquino (1988: I, 85, 2; 76, 2 ad 4; 2008: I, 46, 47, 53). Ver también el interesante artículo de Sanguineti (2011).

19 Maritain (1984: 79-80) afirma: «La connaissance est une opération immanente que procede vitalement de la faculté et est tout entière spécifiée par l'objet-dans laquelle la faculté en s'actuant ellemême est actué par l'objet intentionnelment présent en elle dans sa similitude ou sa species -et qui consiste à devenir immatériellement l'autre lui-même».
} 
cosa entendida, porque no es lo mismo entender la cosa que entender la intención entendida, lo cual hace el entendimiento cuando vuelve sobre su operación; de aquí que unas ciencias traten de las cosas y otras de las intenciones entendidas. Y que la intención entendida tampoco se identifica con nuestro entendimiento, lo vemos porque el ser de ella consiste en su intelección; en cambio, el ser de nuestro entendimiento es distinto de su propio entender (Aquino, 2007: IV, 11).

Con Millán-Puelles (1967: 202) podemos hablar de una irrealidad de la representación en el sentido de que el ser representado consiste en la inmaterialidad de tenerlo presente como objeto de conocimiento. Lo real es el sujeto que conoce y la cosa conocida, pero se requiere de esta irrealidad para acceder a lo real. La representación no suple o sustituye al objeto conocido, sino que remite a él, hace posible o ilumina lo real ${ }^{20}$.

Esta presencia, que muchas veces Tomás explica como representación o existir en de lo conocido en el cognoscente, puede inducir a una lectura en clave representacionista de la filosofía del conocimiento tomasiana. Para obviar esta dificultad, autores como Maritain hacen notar que se podría decir adecuadamente «presentación», en la medida que la relación sujeto-objeto hace presente la cosa misma y, a la vez, remite al entendimiento hacia la cosa (Maritain, 1984: 70-73) ${ }^{21}$. Con el término representación el Aquinate busca dar cuenta de aquello que es lo propio del acto cognoscitivo en el que no hay homogeneidad entre el orden natural o físico y el cognoscitivo, porque al introducirse la co-actualidad de la potencia o forma cognoscitiva y la del objeto conocido, nos situamos en el orden de las operaciones inmanentes. Nos parece que su intención es acentuar tal diferencia.

Si no existiese esta actividad cognoscitiva, tampoco se daría el ser representado. Se deduce, entonces, que el objeto o ser representado, no tiene una existencia propia, un ser extra objetual, sino sólo relativo al sujeto que conoce (Millán-Puelles, 1990: 148-149; 156).

20 Ver también Millán-Puelles (1990: 124). La idea de iluminar lo real es de Polo (1987, II: 190).

21 Maritain no entiende por "presentación» la inmediatez de la presencialidad de la cosa, sino que destaca que ésta no es una inferencia lógica o una deducción a partir de otra instancia diferente a la cosa misma. Es también un término que refuerza la referencialidad o intencionalidad del conocimiento. Ver también Canals (1987: 86-89). 


\section{Conclusión}

Sólo si nos alejamos de la visión especular que hoy se tiene de la representación, contra el que Rorty y muchos filósofos contemporáneos reaccionan, este concepto puede reincorporarse al vocabulario filosófico. La representación no se entiende en Tomás de Aquino como una interferencia entre la inteligencia y la cosa, sino como la necesaria operación y formación que esta facultad ha de realizar para incorporar la realidad en su propio ser de una manera diferente a las afecciones o alteraciones que se explican por la causalidad física.

No es adecuado leer esta expresión tomasiana a la luz de la filosofía de Descartes y de la influencia que este filósofo moderno ejerció. La representación interpretada a la luz del conjunto de la filosofía del conocimiento de Tomás de Aquino, designa la capacidad humana de perfeccionarse a través de la posesión inmanente de la realidad en cuanto conocida. Se refiere a una dimensión que podríamos llamar ontológica, pues se refiere a la condición propia de la persona humana.

Concluimos que en el acto cognoscitivo la representación no es para Tomás de Aquino un estado intermedio porque la misma operación cognoscitiva se realiza en una estrecha colaboración del sujeto con el mundo. Éste se ofrece a la persona en una conexión vital que es el inicio de la operación cognoscitiva. En el caso de la persona, tal conexión no se interrumpe, ni se estanca en ámbitos acotados, sino que le permite ascender y descender, por decirlo de una manera gráfica, desde las elaboraciones abstractas hasta las aplicaciones cotidianas. La representación se puede comprender mejor como una manera mental o intelectual de considerar la realidad - el conocido medium quo y no quodo más claramente aún, la «consideración» misma de lo real, pues en el hombre ésta no puede sino ser intelectual. Es el modo de ser propio del conocimiento humano como tal.

\section{REFERENCIAS}

-Anscombe, G. E. M. \& Geach, P. (1973). Three Philosophers. Oxford: Blackwell. -Aquino, Tomás de (1956). Quodlibet VII. En T. de Aquino: Quaestiones Quodlibetales. (9a edición) Turín: Marietti.

-Aquino, Tomás de (1975-1976). De veritate. Opera Omnia (T. XXII, vol. 1-3).

Roma: Editori di San Tommasso.

-Aquino, Tomás de (1979). Comentarios al "Libro del alma" de Aristóteles. (M. C.

Donadío Maggi de Gandolfi, Trad.) Buenos Aires: Fundación Arché. 
-Aquino, Tomás de (1988). Suma Teológica. (J. Martorell Capó, Trad.) Madrid: BAC.

-Aquino, Tomás de (2008). Suma contra los gentiles. (L. Robles Carcedo y A. Robles Sierra, Trads.) Madrid: BAC.

-Aristoteles (1988). Acerca del alma. (1 $1^{\mathrm{a}}$ edición, $2^{\mathrm{a}}$ reimpresión) (T. Calvo Martínez, Trad.) Madrid: Gredos.

-Aristóteles (1990). Metafísica. (2 edición; V. García Yebra, Trad.) Madrid: Gredos.

-Aristóteles (2008). Categorías. (1ª edición; J. Mittelmann, Trad.) Buenos Aires: Losada.

-Busa, R. (1974). Index Thomisticus. Santi Thomas Aquinatis operum omnium indices et concordantiae. Stuttgart-Bad Cannstatt, Sectio: Frommann-Holzboog.

-Canals, F. (1987). La esencia del conocimiento. Barcelona: PPU.

-Cohen, S. M. (1982). St. Thomas Aquinas on the Inmaterial Reception of Sensible Forms. The Philosophical Review, XCI (2), 193-209.

-Dewan, L. (1984). St. Thomas and the Integration of Knowledge into Being. International Pbilosophical Quarterly, (24), 383-393.

-Hamesse, J. \& Portalupi, E. (1992). Approche lexicographique de l'intentionnalité et de la finalité dans l'oeuvre de Thomas d'Aquin. En J. Follon \& J. McEvoy (Eds). Finalité et Intentionnalité: doctrine thomiste et perspectives modernes, Actes du Colloque de Lowvain-la Neuve et Louvian, 21-23 mai, 1990 (págs. 65-91). Louvain la Nueve et Paris: Editions de L'Institut Supérieur de Philosophie/J. Vrin et editions Peeters, B.P.

-Heidegger, M. (2000). La época de la imagen del mundo. En M. Heidegger, Caminos de Bosque (H. Cortés y A. Leyte, Trads., págs. 63-90). Madrid: Alianza Editorial.

-Hubert, B. (1992). Immatérialité, Intentionnalité et Immanence. Nova et Vetera, (1), 24-47.

-Kenny, A. (1984). Intentionality: Aquinas and Wittgenstein. En A. Kenny, The Legacy of Wittgenstein (págs. 71-76). Oxford: Basil Blackwell.

-Kenny, A. (1989). The Metaphysics of Mind. Oxford: Oxford University Press.

-Kenny, A. (1993). Aquinas on Mind. London and New York: Routledge.

-Llano, A. (1999). El enigma de la representación. Madrid: Síntesis.

-Maritain, J. \& Maritain, R. (1984). Réflexions sur l'intelligence. En J. Maritain \& R. Maritain, Euvres Completes (Vol. III). Fribourg-Paris: Editions Universitaires Fribourg/Editions Saint-Paul.

-Millán-Puelles, A. (1967). La estructura de la subjetividad. Madrid: Rialp.

-Millán-Puelles, A. (1990). Teoría del objeto puro. Madrid: Rialp.

-Moser, R. (2001). Thomas Aquinas, Esse Intentionale, and Cognitive as such. The Review of Metaphysics, (64), 763-788.

-Moya, P. (1999). Dificultades que surgen en la comprensión del conocimiento sensible: una interpretación desde la recepción de Tomás de Aquino del "De anima". Tópicos (16), 87-123.

-Moya, P. (2000). La intencionalidad como elemento clave en la gnoseología del Aquinate. Cuadernos de Anuario Filosófico (105), 1-61. 
-Polo, L. (1987). Curso de teoría el conocimiento. (Vol. I y II; 2ª edición) Pamplona: Eunsa.

-Rorty, R. (1979). Philosophy and the Mirror of Nature. Princeton, N.Y.: Princeton University Press.

-Sanguineti, J. J. (2005). El conocimiento humano. Madrid: Palabra.

-Sanguineti, J. J. (2007). Filosofía de la mente. Madrid: Palabra.

-Sanguineti, J. J. (2011). La especie cognitiva en Tomás de Aquino. Tópicos (40), 63-103.

-Sorabji, R. (1992). Intentionality and Physiological Processes: Aristotle's Theory of Sense-Perception. En M. Nussbaum \& A. Rorty (Ed.), Essays on Aristotle's De anima (págs. 195-225). Oxford: Clarendon Press.

-Vigo, A. (1995). Aristóteles. Física, libros III-IV (Traducción, Introducción, Comentarios) Buenos Aires: Biblos.

Sumario: Introducción; 1. El conocimiento como operación inmanente; 2. La intencionalidad cognoscitiva; 3. La representación; Conclusión; Referencias. 